\title{
Christian Bromberger
}

Professeur à l'Université de Provence - IDEMEC (UMR 6591)

Co-président du Conseil scientifique du Musée des Civilisations de l'Europe et de la Méditerranée (MUCEM)

(1993)

\section{"L’ethnologie de la France et le problème de l'identité"}

Un document produit en version numérique par Jean-Marie Tremblay, bénévole, professeur de sociologie retraité du Cégep de Chicoutimi Courriel: jean-marie tremblay@uqac.ca

Site web pédagogique : http://www.uqac.ca/jmt-sociologue/

Dans le cadre de: "Les classiques des sciences sociales" Une bibliothèque numérique fondée et dirigée par Jean-Marie Tremblay, professeur de sociologie au Cégep de Chicoutimi Site web: http://classiques.uqac.ca/

Une collection développée en collaboration avec la Bibliothèque Paul-Émile-Boulet de l'Université du Québec à Chicoutimi

Site web: http://bibliotheque.uqac.ca/ 


\section{Politique d'utilisation de la bibliothèque des Classiques}

Toute reproduction et rediffusion de nos fichiers est interdite, même avec la mention de leur provenance, sans l'autorisation formelle, écrite, du fondateur des Classiques des sciences sociales, Jean-Marie Tremblay, sociologue.

Les fichiers des Classiques des sciences sociales ne peuvent sans autorisation formelle:

- être hébergés (en fichier ou page web, en totalité ou en partie) sur un serveur autre que celui des Classiques.

- servir de base de travail à un autre fichier modifié ensuite par tout autre moyen (couleur, police, mise en page, extraits, support, etc...),

Les fichiers (.html, .doc, .pdf, .rtf, .jpg, .gif) disponibles sur le site Les Classiques des sciences sociales sont la propriété des Classiques des sciences sociales, un organisme à but non lucratif composé exclusivement de bénévoles.

Ils sont disponibles pour une utilisation intellectuelle et personnelle et, en aucun cas, commerciale. Toute utilisation à des fins commerciales des fichiers sur ce site est strictement interdite et toute rediffusion est également strictement interdite.

L'accès à notre travail est libre et gratuit à tous les utilisateurs. C'est notre mission.

Jean-Marie Tremblay, sociologue

Fondateur et Président-directeur général, LES CLASSIQUES DES SCIENCES SOCIALES. 
Cette édition électronique a été réalisée par Jean-Marie Tremblay, bénévole, professeur de sociologie au Cégep de Chicoutimi à partir de :

Christian Bromberger

“L’ethnologie de la France et le problème de l’identité.”

In Civilisations, Revue internationale d'anthropologie et de sciences humaines, vol. 42, no 2, 1993, pp. 45-63. Numéro intitulé : "En quête d’identité."

[Autorisation formelle accordée par l'auteur le 17 février 2012 de diffuser ce texte dans Les Classiques des sciences sociales.]

gi Courriel : brombergerchristian@gmail.com

Polices de caractères utilisée : Times New Roman, 14 points.

Édition électronique réalisée avec le traitement de textes Microsoft Word 2008 pour Macintosh.

Mise en page sur papier format : LETTRE US, 8.5'” x 11'”.

Édition numérique réalisée le 17 juin 2013 à Chicoutimi, Ville de Saguenay, Québec. 


\section{Christian Bromberger}

Professeur à l'Université de Provence - IDEMEC (UMR 6591)

Co-président du Conseil scientifique du Musée des Civilisations de l'Europe et de la Méditerranée (MUCEM)

\section{"L’ethnologie de la France et le problème de l’identité."}

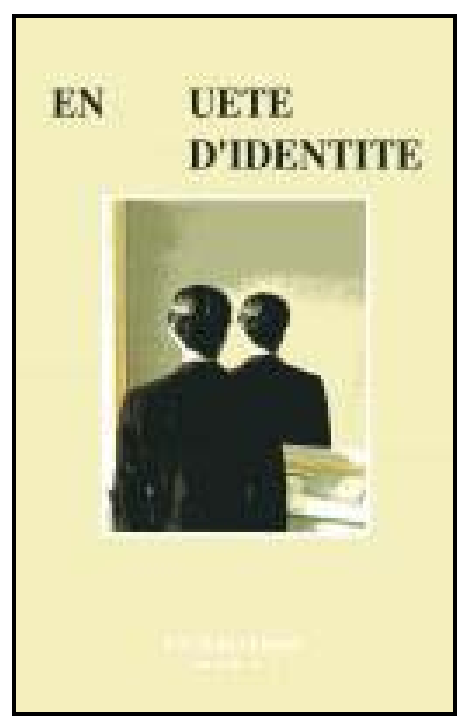

In Civilisations, Revue internationale d'anthropologie et de sciences humaines, vol. 42, no 2, 1993, pp. 45-63. Numéro intitulé : "En quête d’identité.” 


\title{
Table des matières
}

\author{
$\underline{\text { Abstract }}$ \\ L’identité entre excès et soupçons \\ Un contexte historique défavorable à la prise en compte des identités \\ Substantivisme, subjectivisme, contextualisme
}

Bibliographie 
Christian Bromberger

“L’ethnologie de la France et le problème de l’identité.”

In Civilisations, Revue internationale d'anthropologie et de sciences humaines, vol. 42, no 2, 1993, pp. 45-63. Numéro intitulé : "En quête d'identité."

\section{Abstract}

$\underline{\text { Retour à la table des matières }}$

The autor underlines, on the one hand, the interest but also the abuses in the use of the concept of identity in recent ethnological researches. Furthermore, he raises the question of the factors which delayed the taking into consideration of regional and ethnic identities in the work done on France : this desinterest largely results from a specifie political history that favoured a contractuel conception - and not ethnic nor cultural - of the Nation and it is only in period of crisis or of growth, when the references points of collective certitude towards the principle of this project dissolve, that these problems of collectve membership take the first place in the intellectual scene ; thus the contemporary paradox : while the substancial base of cultural differences are declining, social assertion and ethnological studies on identities are the object of an unprecedented interest. Finally, the autor analyses the methods (subtantivist, subjectivist, contextualist), implemented since the institutional emergence of ethnology of France, in order to apprehend the phenomena of identity and tries to bring out the specificities and limits of each approach.

Mots-clés : identité, France, ethnologie française, substantivisme, subjectivisme, contextualisme

Keywords : identity, France, French ethnology, substantivism, subjectivism, contextualism 


\section{L'identité entre excès et soupçons}

$\underline{\text { Retour à la table des matières }}$

Le concept d'identité, d’irruption récente en ethnologie, n'est-il pas un de ces pièges qui jalonnent l'histoire de notre discipline ? Une sorte de fourre-tout, d'ouvre-boîtes universel qui permettrait d'aller vite en besogne en dispensant d'une analyse en profondeur des faits? On éprouve de tels doutes quand on constate, dans les travaux ethnologiques récents, l'extension vertigineuse du concept appliqué généreusement aux objets les plus divers. Tout, ou presque, y devient « identitaire » : non seulement - ce que l'on conçoit bien - les revendications locales, les luttes nationalitaires, la xénophobie mais aussi le vêtement, la cuisine, l'architecture, les savoirs techniques, les espèces animales et végétales, les récits de tradition orale, les manifestations de rue, les rituels ludiques, les fêtes, les révolutions, « la luxation congénitale de la hanche » ${ }^{1}$, les névroses ${ }^{2}$, j’en passe et des bien pires ! On mesure sans peine les risques d'une telle contagion conceptuelle, qui sont essentiellement de deux ordres.

En premier lieu, à se borner à un étiquetage identitaire, distinctif, emblématique des pratiques sociales et culturelles, on aboutit à gommer les propriétés structurelles des phénomènes pour n’en retenir que les manifestations ou les manipulations expressives dans le champ social. Érigée en fonction centrale du faire et du dire, l'identité devient un paresseux principe d'explication et d'interprétation, vidant les pratiques de leur spécificité intrinsèque et de leurs significations entremêlées. Il en est ainsi, par exemple, quand on se contente de proclamer qu'un conte populaire est «identitaire », à l'examen de quelques motifs singuliers ou par référence à l'opinion des auditeurs ;

1 La constatation de ces particularismes génétiques en Bretagne fut à l'origine de la première grande monographie collective et pluridisciplinaire menée sur le terrain français. Entreprise en 1961. Elle eut pour cadre et objet la commune de Plozévet et donna lieu à de nombreux travaux (pour une synthèse voir surtout Burguière, 1975) mettant en oeuvre le concept d’identité jusqu'alors peu utilisé dans le vocabulaire des sciences sociales.

2 On pense à toute une série d'études ethnicisant rapidement divers types de névroses ("névrose bretonne". etc.). 
ne saute-t-on pas alors l'étape essentielle, celle d'une analyse structurale et situationnelle du récit qui en établirait la spécificité morphologique, thématique et contextuelle ? Il en est encore ainsi, pour évoquer un domaine qui m'est cher, quand on réduit l'engouement pour une équipe de football à la simple exaltation d'une appartenance (locale, régionale, nationale). Affirmer une telle banalité - souvent exacte - ne dispense pas de deux interrogations essentielles :

1) sur les valeurs non identitaires que cristallisent les compétitions de football (la performance, l'alliance du mérite individuel et de la solidarité collective, le rôle de la chance, de la tricherie, d'une justice parfois arbitraire sur le chemin du succès, etc.), valeurs qui forment la trame symbolique de la réussite dans le monde contemporain ${ }^{3}$ et qui sont sans doute au principe de cet engouement planétaire ;

2) sur les modalités selon lesquelles des histoires individuelles et collectives (celles de groupes et de réseaux sociaux, de quartiers, de villes, de régions) se croisent avec l'histoire répétitive et singulière tout à la fois qui se déroule sur le terrain (en examinant comment le style de jeu de l'équipe, plus ou moins rigoureux, plus ou moins flamboyant, est perçu comme la métaphore d'un mode spécifique d'existence collective ou encore comment une alternance de déboires et de victoires est conçue comme la figuration d'un destin, bref en tentant de cerner les liens de motivation entre contenus de l'identité et formes du jeu).

À se cantonner dans le simple repérage de fonctions identitaires, on tomberait, au bout du compte, dans le même travers que ces amateurs qui privilégient la marque ou la signature, non l'objet ou le tableau.

En second lieu, à qualifier invariablement d' "identitaires » les objets les plus hétérogènes, on risque de placer sur le même plan des faits d'inégale intensité dans le champ social : le port d'une coiffe et

3 Sur le football comme "jeu profond" voir Ehrenberg (1991) et Bromberger (1992b). 
le racisme ordinaire, l'étiquette d'un fromage, vantant les qualités d'un terroir, et une lutte nationalitaire. Toutes ces pratiques relèvent sans doute de l'expression, de la promotion ou de la revendication d'une identité mais, à les subsumer sous ce concept, on tend à en niveler les significations disparates, à en raboter les aspérités, à les plonger « dans une nuit où tous les chats sont gris ». C'est bien là le second piège qui guette l'usage et l'abus de la notion.

Faut-il, pour autant, « Jeter » l’identité « avec l'eau du bain » ?

En fait, ce concept, avec les vertus de ses vices, est venu combler utilement une lacune dans le vocabulaire des sciences sociales : il invite à renouer les fils de l'expérience et de la conscience collectives que l'analyse souvent disjoint, à repérer des principes unificateurs au sein de couches sociales ou de groupes localisés, bref à mettre en oeuvre une démarche synthétique. Comme de l'alcool, on doit donc en faire un usage modéré mais on ne peut guère s'en passer.

Y a-t-il eu une manière proprement française de poser le problème des identités, qu'elles soient territoriales, sociales ou ethniques ? Quels modes d'approche a-t-on mis en oeuvre pour traquer ces phénomènes qui ne sont pas des objets empiriques, comme peuvent l'être un conte, un tournevis, une loi, un mariage, etc. ? Comment rendre compte enfin du paradoxe qui caractérise la situation française contemporaine où les affiliations collectives sont de plus en plus brouillées, où le socle substantiel des différences culturelles s'étiole et où, pourtant, l'affirmation sociale et l'étude ethnologique des identités ont rarement connu pareille vogue?

\section{Un contexte historique défavorable à la prise en compte des identités}

$\underline{\text { Retour à la table des matières }}$

La France offre une configuration culturelle qui, par sa diversité, aurait dû aiguiser l'appétit des folkloristes puis des ethnologues et susciter réflexions, théories, débats sur les faits d'identité régionale ou ethnique: on y parle traditionnellement plusieurs langues distinctes (neuf au total, dont cinq gallo-romanes); de l'Alsace au Pays basque, 
de la Bretagne à la Provence, le clavier des différences culturelles est particulièrement contrasté ; c'est, par ailleurs, une vieille terre d'immigration où se sont fondus les apports les plus divers. Or, paradoxalement, le constat et l'affirmation de ces différences n’ont guère préoccupé, jusqu’à un proche passé, les élites intellectuelles nationales, comme ce fut le cas dans d'autres pays européens. Une multitude d'indices témoigne de ce désintérêt, qui contraste, toutes proportions gardées, avec la fringale ethnographique précoce qu'a connue l'Europe centrale et orientale : le statut marginal de l'ethnologie de la France dans le champ universitaire (rien de comparable aux chaires de Volkskunde dans les pays germaniques et l'on ne saurait simplement attribuer cette marginalisation au développement, autrement plus prestigieux, dans notre pays, de l'ethnologie exotique) ; le développement très inégal des musées des Beaux-Arts, fleurons nationaux, et des musées d'ethnographie, infiniment moins soutenus par les pouvoirs publics (Cuisenier, 1991); l'absence d'atlas ethnographique de la France, quand chaque peuple ou nation s'en est doté en Europe du nord, du centre et de l'est, souvent dans des contextes de construction, d'humiliation ou de revendication étatiques ${ }^{4}$; la faible empreinte du romantisme sur l'émergence de l'ethnologie de la France : au panthéon des fondateurs aucune figure comparable à celles des frères Grimm en Allemagne (de façon révélatrice ce sont les préfets qui, au début du 19ème siècle, ont entrepris des enquêtes systématiques, commandées d'en haut, sur les moeurs et les coutumes des populations qu'ils administraient) ; à la même époque peu de réflexion philosophique s'interrogeant pleinement sur la diversité des cultures à la mesure d'un Herder (quelles que soient, par ailleurs, les limites de cette pensée) et, plus près de nous, aucun théoricien de l'envergure de Gramsci ayant pris pour objet les relations entre cultures populaires et culture savante. La littérature est aussi un bon baromètre des intérêts majeurs d'une société : peu de grands écrivains français des 19ème et 20ème siècles ont associé leur écriture à des prosopopées régionales ou puisé leur inspiration dans le génie ethnographique des lieux. La carte dressée par Roland Dorgelès au début du siècle, où à chaque province correspond l'écrivain qui en est le chantre, et le recensement des auteurs régionalistes établi par A.-M. Thiesse $(1988,1991)$ sont

4 Sur les contextes idéologiques favorables à l'élaboration d'atlas ethnologiques, voir Bromberger, Dossetto et Schippers (1982-1983). 
involontairement sévères : à quelques exceptions près, n’y figurent que des auteurs mineurs. Partant de ce constat, A. Billy pouvait proclamer, dans un numéro du journal Le Figaro en 1946 : "La valeur dominante de la littérature française est l' universalisme » 5 .

Au fond ce type de préoccupations pour les cultures et les particularismes régionaux ne semble occuper le devant de la scène intellectuelle française qu'en période de crise ou de restauration, quand se rompent les équilibres économiques et démographiques ou encore quand la Nation échappe aux fondements modernes de son histoire, tels que les ont conçus les Lumières et incarnés la Révolution : sous la Restauration, où l'on se passionne pour la détection des survivances celtes; dans la seconde moitié du 19ème siècle où, sous le coup de l'exode rural, se créent, dans plusieurs provinces, des mouvements régionalistes de sauvegarde et défense de cultures en déclin ; dans les années 1930 où l'enracinement provincial s’offre comme une alternative à la crise, au dépeuplement des campagnes et comme un facteur de paix sociale (il s'agit alors plutôt d'odes à la diversité des terroirs que de revendications régionales, sauf dans quelques cas particuliers comme l'Alsace, voir infra ) ; sous le régime de Vichy, où sont célébrés et promus les langues locales et le folklore ${ }^{6}$; dans les années 1970 où, sur une toile de fond radicalement différente, tissée dans le prolongement des événements de mai 1968, fleurissent la revendication régionale, écologiste, les associations locales, les aspirations à la réappropriation des identités territoriales (c’est précisément dans ce contexte fébrile, enthousiaste, contestataire que le concept d'identité collective fait une irruption massive dans le vocabulaire des sciences sociales puis dans celui de politiciens et d'aménageurs); au fil des années 1980 enfin où le débat public, prenant pour cible l'étranger, se noue autour des différences culturelles dans une société qui a perdu, j’y reviendrai, les repères de certitude collective qui en fondaient l'unité.

Ce désintérêt de l'intelligentsia pour les identités des régions et des « peuples » réunis sur le territoire national résulte largement d'une

5 Cité par A. -M. Thiesse (1988 : 231).

6 On trouvera un ensemble de données sur l'exaltation des valeurs provinciales et sur la politique ethnologique menée sous le régime de Vichy dans Faure (1989). 
histoire politique originale qui a promu une conception contractuelle et non ethnique ou culturelle - de la nation. Comme l'écrit excellemment D. Schnapper (1991: 71), à la suite d'E. Renan ${ }^{7}$ et de F. Sieburg ${ }^{8}$, "la nation française a été une forme politique qui a transcendé les différences entre les populations, qu'il s'agisse des différences d'origine sociale, religieuse, régionale ou nationale et les a intégrées en une entité organisée autour d'un projet politique commun ». Contrairement à la conception allemande fondant l'appartenance nationale sur les liens du sang, le paradigme français prend en compte le droit du sol Vus soli) dans l'acquisition de la nationalité et valorise l'adhésion individuelle et volontaire à la citoyenneté. " On entre dans la nation française comme dans une communauté religieuse constituée non par le sang mais par l'esprit ", notait F. Sieburg. Et D. Schnapper rappelle justement que, dans la vie politique française, il n'y a jamais eu de représentation politique de groupes ethniques et que, lors des naturalisations, les étrangers sont saisis comme individus, non comme membres d'une communauté (à ce titre les associations d'originaires ne jouent aucun rôle dans ce processus, contrairement à ce qui se passe aux États-Unis par exemple).

Qui voudrait se donner une idée exemplaire et rapide de ce melting-pot à la française, de la tradition républicaine d'intégration n'aurait de meilleur moyen que de consulter la composition des équipes nationales de football qui se sont illustrées pendant la dernière décennie. L'équipe qui, par exemple, rencontrait la Yougoslavie le 16 décembre 1985 "était emmenée par Michel Platini, petit-fils d'Italien. Elle comprenait. dans ses lignes arrières, Battiston (petitfils d'Italien lui aussi), Manuel Amoros (fils d'Espagnol), William Ayache ("pied-noir» venu d'Algérie). En milieu de terrain, à part Platini, on trouvait Giresse (fils d'immigré espagnol par sa mère), Tigana (né au Mali, arrivé enfant à Marseille), Luis Femandez (né en Espagne et immigré en France avec sa famille). La ligne d'avants comptait José Touré (fils d'un immigré malien lui aussi), Yannick Stopyra (petit-fils de Polonais) » ${ }^{9}$. Dans ce processus d’intégration et

7 On sait que Renan (1947, rééd.) est le penseur d'une conception volontariste de l’appartenance nationale fondée sur "le vouloir vivre ensemble" et "le plébiscite de tous les jours".

8 Dans son célèbre essai Dieu est-il français ?

9 Voir l'étude de Beaud et Noiriel (1990) sur football et immigration. 
de « fabrications des Français » ${ }^{10}$, on sait le rôle important qu'a tenu l'école publique laïque visant à effacer, idéalement au moins, les différences d'origines régionales et nationales et à unir les futurs citoyens autour d'un projet républicain commun (le port de la blouse, la stigmatisation des patois et l'imposition d'une norme linguistique, l'élaboration d'un folklore républicain, etc., ont été autant d'expressions de ce souci d'assimilation et d'unification). On conçoit sans peine que, dans un tel contexte où s'affiche l'arrogance de l'universel, l'étude des identités n'ait pas trouvé un terreau intellectuel favorable. La France moderne, jacobine s'est construite contre les peuples - aux sens germanique et stalinien du terme-, contre les particularismes ou le folklore qui fondaient ailleurs l'idée de nation. Une telle vision politique, postulant la primauté de l'État, l'égalité abstraite des individus et non leur enracinement communautaire, a eu des effets sensibles sur le fonctionnement de la société française : l'absence, dans les grandes agglomérations, de "ghettos ethniques » s'autogérant selon des normes propres (comme en Angleterre et aux États-Unis par exemple), le cantonnement de l'expression des différences dans la sphère privée (une sorte de jardin secret cultivé avec soin) et longtemps la faiblesse des revendications minoritaires.

Or, paradoxalement, c’est au moment où les identités régionales et ethniques perdent de leur substance qu'elles s'affirment bruyamment sur la scène publique. Au fond, jamais un Corse, au fil de l'histoire, n'a ressemblé autant à un Breton ou à un Parisien, et pourtant jamais il n'a proclamé plus haut sa différence. À une toute autre échelle et sur un mode mineur, jamais les habitants des « pays » de France, c'est-àdire des microrégions, souvent héritières des pagi gallo-romains, n’ont eu des pratiques culturelles aussi homogènes - bien que toujours diverses - et jamais pourtant les " pays » n’ont été autant à l’honneur, blasonnant les produits locaux, affirmant leur spécificité culturelle à travers ces institutions originales que sont les écomusées où se réfléchissent territoire, mémoire et projet collectifs ${ }^{11}$. Paradoxe supplémentaire - qui souligne l'écart entre l'identité substantielle, que l'on constate, et l'identité performative, qui se dit, - les appartenances bruyantes célèbrent souvent, dans notre société, des groupes défunts

10 Selon l'expression de M. Agulhon (1989).

11 Sur la genèse de ces institutions, leur place particulière dans l'ensemble des musées voir Bromberger (1992a). 
ou à l'agonie dont le substrat s'étiole ; les groupes vivants, alertes, heureux - s'il en existe - n'exigent, en général, pas un tel supplément d’âme. La réalité suffit à prouver leur dynamisme singulier. L’identité qui s'affiche est souvent, en revanche, l'écho d'une catastrophe (disparition d'une activité, crise économique locale, etc.). Autrement dit on pourrait opposer, comme le suggère G. Ravis-Giordani (1989), des identités sans peuples à des peuples sans identités ou encore souligner, avec M. Agulhon (1989 : 120), « que, en 1914, les Français étaient unis dans la conscience nationale, et divers dans les comportements culturels, alors qu'aujourd'hui ils sont divers et divisés par rapport au National mais plus unis dans les comportements culturels ». Les raisons de cette situation paradoxale sont multiples mais son émergence, dans les années 1960-1970, tient à la dissolution dans le corps social des repères de certitude collective qui alimentaient le projet républicain et produisaient des normes d'insertion acceptées par tous. $\mathrm{Au}$ triomphalisme de l'unité nationale, qui réunissait la droite et la gauche, se substitue alors une multitude de discours dénonçant le colonialisme intérieur, revendiquant le droit à la différence, l'autonomie territoriale, etc., bref mettant en cause ce que l'on a appelé «l'exception française ». Cette tendance à la proclamation des appartenances sera renforcée et infléchie tout à la fois, au tournant des années 1980, par l'effondrement des idéologies universalistes qui relativisaient les affirmations des identités bio-sociales, ethniques ou régionales. Dans le vacuum s'imposent d'autant plus facilement des catégories d'affiliation immédiates : la région, l'ethnie, la classe d’âge, etc., qui investissent l'espace laissé libre par les grandes ou les mauvaises causes.

Sur cette toile de fond mouvante, quelles furent les démarches mises en oeuvre par l'ethnologie de la France pour traquer différences et identités? 


\section{Substantivisme, subjectivisme, contextualisme}

$\underline{\text { Retour à la table des matières }}$

On peut grosso modo - et quitte à caricaturer - distinguer quatre modes d'appréhension des phénomènes d'identité, qui se sont chevauchés plutôt que relayés, depuis l'avènement formel et institutionnel de l'ethnologie de la France, que symbolise la création, en 1937, du Musée National des Arts et Traditions Populaires.

Jusqu'aux années 1950 domine une démarche réaliste, substantiviste, souvent historiciste qui vise à typer de larges espaces culturels et éventuellement à en expliquer la formation. C'est le temps des grandes enquêtes extensives, de la constitution de corpus sur le folklore enfantin, l'alimentation, les moyens de transport traditionnels, l'architecture rurale, le mobilier régional, etc. ${ }^{12}$ Il ne s'agit guère alors de disserter sur l'identité ou sur les sentiments d'appartenance mais d'analyser des discontinuités culturelles à travers des objets ou des pratiques. Ces recherches, adoptant un point de vue que l'on appellerait aujourd'hui etic, visent, en effet, à constituer un savoir cumulatif d'ordre aréologique. De façon symptomatique les seuls résultats d'un certain nombre de ces grands chantiers, auxquels participent des géographes, sont des cartes ethnographiques 13 . On souhaite alors scruter, à travers ces procédures à grande échelle, la fragmentation du paysage culturel de la France et, en particulier, les contrastes entre le nord et le sud, entre les mondes d'oïl et d'oc ou encore la continuité de "zones folkloriques », selon l'expression de Van Gennep. ${ }^{14} \mathrm{La}$ plupart de ces données aréologiques sont regroupées dans l’ouvrage de F. Krüger (Géographie des traditions populaires en France), dernier vestige de cet engouement pour les grandes enquêtes exten-

12 Pour un inventaire et une présentation de ces différentes enquêtes voir Cuisenier (1982-1983).

13 On trouvera un inventaire à la fois descriptif et critique de ces documents dans Cuisenier (1982-1983), Bromberger, Dossetto et Schippers (1982-1983). La plupart de ces cartes sont regroupées dans l’ouvrage de Krüger (1950).

14 Sur la notion de "zone folklorique" chez Van Gennep voir Belmont (1981). 
sives 15 ... qui remonte à 1950 . Depuis, on s'est contenté de systématiser ces collectes déjà anciennes et les inventaires raisonnés de pratiques, de techniques, de contes ... ont rarement dépassé le cadre des anciennes provinces ${ }^{16}$. Les seules initiatives d'ampleur nationale, visant à faire ressortir des différenciations à grande échelle, ont été l'oeuvre de dialectologues, de sociologues ou encore d'historiens ${ }^{17}$. Mais dans tous ces travaux, qu'ils émanent de l'ethnologie ou des disciplines connexes, la problématique de l'affirmation et de l'expression des identités apparaît bien marginale ; il s'agit de repérer des différences, d'en instruire l'histoire et la géographie, non pas de s'attarder sur la conscience et la mémoire collectives des acteurs. Les oeuvres visant à définir, voire à exalter, les " génies » régionaux proviennent, à l'époque, d'horizons très divers : soit de cercles à dominante poétique (tels Les Cahiers du Sud consacrant en 1943 un numéro au " génie d'oc » promu en symbole de l'humanisme face à la force et à la haine), soit de sociétés savantes (sur le cas particulier de l'Alsace où celles-ci militent pour la reconnaissance d'une culture indigène Heimatliche Kultur - germanique, voir Raphaël et Herberich-Marx, 1991) ; soit d'érudits locaux confortés dans leurs prosopopées provinciales par l’idéologie du régime de Vichy.

Au tournant des années 1950, la monographie de commune devient le genre dominant éclipsant les grands programmes documentaires. Ce changement radical de perspective et d'échelle tient à plusieurs facteurs - pratiques et théoriques - dont l'importance respective est difficile à évaluer. D’une part, la pratique directe et prolongée du terrain, nouveauté de l'après-guerre, limitait le champ de l'observation : «Levillage, écrivait M. Maget (1955: 380), est un lieu de prédilection pour l'enquête monographique. Il n'a pas, en effet, un volume tel qu'il

15 Pour de plus amples développements sur la portée de ce revirement méthodologique voir Bromberger (1987).

16 Voir, à titre d'exemple, l'oeuvre de J. -M. Guilcher, notamment son travail sur la danse en Béarn et Pays Basque (1984).

17 On pense aussi bien aux Atlas linguistiques et ethnographiques réalisés dans le cadre de l'Atlas linguistique de la France (ALF) qu'aux travaux des historiens sur la répartition des différents types de coutumes successorales ou des sociologues sur la diversité de la pratique religieuse selon les régions de France (voir, par exemple, Isambert et Terrenoire, 1980). Après s’être complètement désintéressée de ces travaux extensifs, l'ethnologie de la France semble timidement y reprendre goût depuis quelques années. 
dépasse les capacités d'absorption d'un seul chercheur qui, même en cas de spécialisation, peut tenir une vue synoptique individualisante de l'ensemble du groupe "; d'autre part, sous l'influence de l'anthropologie anglo-saxonne et par un rapprochement de l'ethnologie exotique et de l'ethnologie de la France, les thèmes de recherche et les intérêts théoriques se déplaçaient : l'organisation économique, sociale et familiale, les institutions politiques ... faisaient irruption sur le devant de la scène ethnologique et l'on voyait mal où les saisir sinon à travers des études de cas approfondies. Par ailleurs, le structuralisme et le fonctionnalisme ambiants postulaient non plus des inventaires par séries analytiques mais une approche holistique, totalisante - la fameuse "community asa whole » de R. Redfield (1960 : 149-168) - faisant ressortir les interactions entre les éléments constitutifs d'une société ${ }^{18}$. D'abord exclusivement rurale, centrée sur des communes ou sur des "pays ", la démarche monographique diversifia progressivement son horizon : quartiers de villes, entreprises artisanales puis industrielles firent l'objet d'une même attention descriptive.

Dans un premier temps, tous ces travaux visaient à analyser le fonctionnement de ces collectivités, à en typer la spécificité par une appréhension serrée des différents secteurs de la vie sociale. La grande étude collective sur l'Aubrac - entreprise en 1964 et dont la publication fut achevée en 1982 - témoigne de ce souci d'exhaustivité objectiviste qui plane sur la recherche jusqu'au tournant des années 1970.

La démarche s'infléchit alors sensiblement; ce n’est plus tant le socle substantiel des identités que l'on vise à mettre au jour mais les sentiments et les expressions des appartenances ; à l'analyse pointilleuse des indicateurs traits de différenciation et d'identification que révèle une étude substantive et contrastive des faits - se substitue la recherche des marqueurs emblématiques, c'est-à-dire des traits reconnus et retenus, par les acteurs eux-mêmes, comme symboles d'identité et d'altérité. On mesurera l'étendue de ce revirement en examinant ces deux définitions successives de la notion de patrimoine ethnologique, englobant, dans le premier cas, «les modes spécifiques d'existence

18 Sur les différents facteurs qui ont entraîné cet engouement pour la monographie de communauté voir Bromberger (1987). 
matérielle et d'organisation sociale des groupes humains, leurs savoirs, leur représentation du monde et, de façon générale, les éléments qui fondent l'identité de chaque groupe et le différencient des autres » 19 et, dans le second cas, les seuls objets dont « le groupe social a la conscience intime qu'ils font partie de son patrimoine » 20 . Cette approche subjectiviste, emic, a connu ses «minimalistes » et ses « maximalistes » : les premiers se sont intéressés aux expressions des appartenances (le blason, les noms individuels et collectifs, les comportements d'identité à usage interne ou externe, les fêtes, les pratiques ludiques, la mémoire locale, etc.) sans négliger l'observation du soubassement de ces faits ; les seconds ont valorisé la parole au détriment de l'observation et fait la part belle au discours de l'Autre sur lui-même. Cette dernière tendance a trouvé un contexte particulièrement favorable à son épanouissement dans le contexte idéologique des années 1970 ; les aspirations à la convivialité, à l'associationnisme, à la citoyenneté de proximité, au retour à la vie villageoise, la nostalgie de " ce monde que nous avons perdu » donnaient à tout témoignage vécu sur la vie locale autrefois un prix - affectif et souvent commercial - particulier. Ces aspirations, convergeant avec les revendications de certains mouvements régionalistes, se traduisirent non seulement par une fringale ethnographique (les quelques 800 créations ou recréations de musées que l'on pourrait, pour la plupart, qualifier de musées d'identité en fournissent un éclatant symptôme) mais aussi par l'émergence de l'endo-ethnologie (do it yourselfl), tendance qui trouva des échos favorables chez certains professionnels, sensibles à cette "rébellion des objets ethnologiques ", selon l'expression d'A. M. Cirese. Ces professionnels dénonçaient volontiers « la triste insuffisance de l'intelligible face à la somptuosité débordante du fait brut »et plaidaient " pour la réappropriation de l'analyse sociale par les acteurs eux-mêmes ». L'identité est alors une valeur sûre dont une partie de la littérature ethnologique et para-ethnologique colporte une vision enchantée.

Vient ensuite l'ère du soupçon : à l'exaltation complice des identités se substituent des interrogations sourcilleuses sur la production des identités. On dénie volontiers toute réalité stable et substantielle aux

19 Sur cette définition du patrimoine ethnologique élaboré en 1979, ses origines, ses implications voir Chiva (1990).

20 Selon l'expression d'Ollivier (1987 : 127). 
affirmations et expressions identitaires et l'on traque, à travers cette mise en scène spectaculaire des appartenances, les stratégies de groupes qui s'affrontent sur la scène sociale. À l'enchantement succèdent le désenchantement, le scepticisme, le regard critique. Aux gesticulateurs de l'identité se substituent les rabat-joie. Faut-il mettre en parallèle l'émergence de cette nouvelle attitude et le déclin du discours prônant « le droit à la différence » communautaire, qui s’efface progressivement devant des propositions plus nuancées réclamant « le droit à l'indifférence » ? En tout cas, la situation brouillée des années 1980, balançant entre "différentialisme » et "pluriculturalisme », d'une part, et « intégrationnisme » de l'autre, a été profondément déconcertante, au premier chef pour ceux qu'elle visait, et a invité à considérer avec une prudente distance le recours au concept d'identité. Puisque les uns - qui discourent et promulguent - et les autres - qui subissent et revendiquent - peuvent tantôt poser les identités comme irréductibles tantôt comme malléables et secondaires, il y a bien lieu de s'attarder sur les contextes de ces affirmations contradictoires. Si les identités ont un contenu mouvant, sont tantôt vives tantôt dormantes, tantôt imprescriptibles tantôt négociables, faut-il en faire si grand cas ou bien s'interroger d'abord, avec une moue sceptique, sur ce qu'elles recouvrent et dissimulent?

Les arguments ne manquent certes pas à l'appui d'une vision distanciée, voire critique, des identités. Faisons rapidement le tour des problèmes soulevés.

On a d'abord souligné, à juste titre, que les identités ne sont jamais monolithiques - a fortiori dans une société complexe - mais multiples, enchevêtrées, variables selon les statuts sociaux et les contextes d'interaction. M. Bozon et A. -M. Thiesse (1985: 13-14) montrent, par exemple, que les habitants du Valois revendiquent des appartenances différentes selon leurs trajectoires respectives : les grands cultivateurs et les néo-résidents fortunés tiennent au rattachement du Valois à l'Île-de-France qui connote la tradition du domaine royal et le rayonnement de la prestigieuse capitale; en revanche, "les quelques nouveaux installés de milieu populaire », marqués par une expérience résidentielle difficile en banlieue parisienne, "se réfèrent exclusivement au rattachement administratif actuel de la région à la Picardie ». 
Sur le même chemin critique, on a fait remarquer que l'affirmation des appartenances territoriales ou ethniques éclipse des affiliations plus discrètes mais plus décisives - à des réseaux sociaux fondés sur des rapports d'amitié, d'intérêt, de solidarité diverse ${ }^{21}$. Bref le paradigme de la limite - villageoise, régionale, ethnique - doit souvent céder le pas à celui du réseau - translocal ou transcommunautaire - qui s'affranchit. partiellement au moins, des statuts acquis par la naissance ou par la résidence.

Contre un substantivisme étroit, on a aussi remis en cause l'adéquation, parfois postulée, entre sentiments d'appartenance et spécificités culturelles ou, pour dire autrement les choses, entre cartes d'identité et cartes ethnographiques. Faut-il le souligner ? Les régions culturelles que met au jour l'ethnologue par un examen serré des indicateurs ne correspondent souvent pas aux espaces d'appartenance que revendiquent et auxquels s'identifient les individus. Par exemple, le Rhône représente une frontière beaucoup plus forte sur le plan des représentations que sur celui des usages, dans l'ensemble homogènes de part et d'autre du fleuve qui sépare la Provence occidentale du Languedoc oriental ; dans la vallée de Barcelonnette (qui confine avec l'Italie) les habitants se sentent fort différents de leurs voisins piémontais qui partagent pourtant un même substrat d'identité (une langue et des techniques culturales communes entre autres). Bref, les traits culturels se déploient par vastes nappes qui débordent généralement les espaces perçus et conçus par les hommes comme des territoires emblématiques.

Ce contraste entre identité substantielle et identité performative se lit encore à travers un paradoxe que nous avons déjà souligné : les mouvements de revendication identitaire ne sont pas de simples caisses de résonance de différences culturelles (plus il y aurait de différences, plus il y aurait de revendications) mais émanent le plus souvent (on ne saurait ici encore moins qu'ailleurs généraliser) de groupes qui, d'une part, se sentent malheureux, victimes, dépossédés et, d'autre part, ont les moyens institutionnels ou tactiques de le faire savoir (les exclus n’ont guère la possibilité de prendre la parole). Au fond les Bourguignons et les Rhône-Alpins se soucient beaucoup moins de proclamer hautement leur identité que les Marseillais ou les

21 Voir Bromberger (1987 : 83). 
Corses chez qui sévissent la crise, le sous-emploi, la dépopulation. Dans des sociétés fondées sur le succès, les derniers de la classe - les appartenances n'étant pas seulement des moyens de classification relativiste mais des principes de classement hiérarchique - s'insurgent contre leur position et en attribuent la responsabilité - pas toujours à tort - aux Autres. Mais les Catalans et les Lombards, arguera-t-on. Voilà des sociétés prospères, premières de leur classe, qui clament pourtant haut et fort leur identité. Sans entrer dans le détail d’histoires complexes, remarquons qu'il s'agit ici de manifestations réactionnelles contre l'inadéquation entre pouvoir économique et pouvoir politique (cas de la Catalogne) ou encore contre les charges que fait peser le sud, moins développé, sur le nord du pays (cas de la Lombardie). Il serait sans doute absurde de réduire le sens de ces mouvements à de simples revendications politiques et économiques ; notons seulement que l'affirmation bruyante de l'identité va souvent de pair, y compris chez les premiers de la classe, avec le sentiment - fondé ou infondé - d'une injustice qu'il faut réparer.

En poursuivant sur ce chemin soupçonneux, on aboutirait à une vision purement instrumentale de l'identité, où discours, emblèmes, professions de foi culturelles ne seraient, au bout du compte, que des parures factices, recouvrant d'autres enjeux, y compris les ambitions de Rastignac-s déçus ou conquérants. Et dans cette mise en scène, l'ethnologie tiendrait, si elle n'y prenait garde, un curieux rôle, codifiant, comme naguère le folklore, d'anciennes coutumes "spécifiques » à travers textes et musées, contribuant à élaborer des outils identitaires et prêtant le flanc aux querelles entre Rastignac-s locaux qui s'affrontent pour détenir l'autorité légitime de dire l'identité au nom des autres.

On est fondé à éprouver de tels soupçons contre les visions fixistes et culturalistes des identités quand on constate avec quels facilité et entregent les sociétés changent rapidement, dans ce domaine, leur fusil d'épaule. Les chasseurs du nord de la Vénétie fournissent, à point nommé, un exemple coruscant de ce genre de revirement. S'adonnant naguère à leur passion sur un mode "latin ", festif, bruyant, insouciant, les voici désormais qui tiennent un discours "germanique », gestionnaire, écologiste sur la faune et prônent une chasse sportive, sélective, silencieuse ; symbole de cette " conversion collective », ils ont adopté, au nom d'une tradition inventée récemment, la veste, le 
chapeau tyroliens et « d'autres gadgets d'origine autrichienne » (Dalla Bernadina, 1989). Ce changement d'identité apparaît, au bout du compte, comme une réponse, contextuellement appropriée, à la remise en cause, par les associations écologistes, de la pratique de la chasse. Après tout, le plaisir d'un civet de lièvre ou d'une brochette de petits oiseaux vaut bien que l'on porte un chapeau tyrolien et que l'on tienne un discours écologiste! L’identité régionale présente parfois les mêmes aptitudes surprenantes à la métamorphose de sa filiation et de ses apparences. Ainsi le mouvement régionaliste alsacien a balancé, au cours de son histoire, entre " germanisme » et " francisme » (Denis, 1987 ; Raphaël et Herberich-Marx, 1991). Avant l'annexion de 1870 domine un groupe de lettrés luttant «pour la conservation de la langue, de la culture, des us et coutumes germaniques ». Pendant la première annexion, l'équipe qui dirige et anime le Musée alsacien de Strasbourg (créé en 1902) "cherche »au contraire, "à démontrer la spécificité de la culture populaire alsacienne par rapport aux pays germaniques et ses affinités avec la France » (Denis, 1987 : 9). Entre les deux guerres, " on assiste à un retournement de situation puisqu'il s'agit alors, pour l'Alsace, d'affirmer son identité face à la France, beaucoup plus centralisatrice que l'empire allemand » (ibid : 10); on exalte donc le Volkstum - génie ethnique - alsacien et la Muttersprache - langue maternelle - allemande (Raphaël et Herberich-Marx, 1991 : 394-395). Bref les revendications d'identité culturelle ont fluctué au rythme des vicissitudes historiques, puisant leurs arguments tantôt dans la tradition germanique, tantôt dans la tradition française, pour asseoir la reconnaissance de la "petite patrie ». Si le visage d'une identité change selon le contexte politique, il présente aussi des traits bien différents à l'échelle d'une collectivité où s'affrontent des groupes d'obédience diverse. L'organisation et la célébration des fêtes locales sont souvent de puissants révélateurs de ces enjeux conflictuels que cristallise l'énonciation de l'identité. F. Lautman (1988) montre ainsi comment la fête locale de Sainte Estelle, telle qu'elle se déroule à Saint-Junien, dans le Limousin, en 1985, donne lieu à des processions concurrentes, placées sous l'égide de la même patronne mais aux significations opposées l'une, organisée par la municipalité ... communiste, célèbre l'identité de la ville, sa continuité historique où les édiles locaux aiment ancrer leur action pour mieux asseoir leur pouvoir ; l'autre regroupe des Félibres - dont Sainte Estelle est aussi la 
patronne - et clame, sur un mode droitier, son attachement à la maintenance de la culture d'oc.

Faut-il, en définitive, s'arrêter à cette vision désenchantée de l'identité, superbe château de cartes aux apparences trompeuses, et, du coup, réduire le rôle de l'ethnologue à celui d'un Zorro dénonçant des impostures ? Ce serait, à l'évidence, tomber d'un excès dans l'autre. Que l'identité nous parle, à travers les textes et les emblèmes qui la célèbrent, d'enjeux, de conflits, d'ambitions, de rancoeurs, c'est un fait désormais suffisamment établi. Mais ces "manipulations » ne s'exercent que dans un champ étroit de références, délimité par des pratiques communes, des symboles ancrés dans une expérience collective et façonnés par la durée. Autrement dit, les traits de culture, le langage identitaire ne peuvent «fonctionner » comme pôles de reconnaissance, voire de mobilisation, que s’ils s'inscrivent dans une mémoire vive ou dormante, dans une histoire qui était déjà là avant nous, les passants, ou encore dans un projet consensuel préexistant. C'est là la condition de leur crédibilité, sauf à poser que les hommes sont des « idiots culturels ", incapables de distance critique et, partant, des proies faciles pour des marchés identitaires de dupes. L'identité, si elle est mouvante, n'est pas pour autant arbitraire ; elle ne se décrète ni ne s' " invente » jamais complètement. Voilà des propositions qui semblent infirmées par les comportements de nos chasseurs du Veneto ou des militants de la cause alsacienne, dont nous pointions plus haut les fluctuations identitaires. Mais revenons un instant sur ces cas extrêmes où la coutume change si rapidement de costume ou d'idiome. En fait des modifications aussi spectaculaires ne sont possibles et crédibles que dans des régions-frontières qui offrent à portée du regard et de l'expérience quotidienne des modèles opposés de pratiques. Autrement dit, on imagine mal un chasseur calabrais endosser avec autant de célérité la tenue du sportsman tyrolien... Remarquons dans le même sens que l' ' 'invention', des traditions est rarement un jeu qui s'effectue ex nihilo : les treize desserts du repas de la veille de Noël, le costume du gardian (pasteur de taureaux), perçus aujourd'hui comme des emblèmes provençaux de très grande profondeur historique, sont, en fait, des innovations codifiées par les folkloristes au début du 20ème siècle mais ces «traditions » n’ont pu s’imposer avec autant de fortune que parce qu'elles apparaissaient comme des remodelages crédibles de coutumes déjà existantes (sur la fixation des 
treize desserts, voir Bromberger 1989: 227 et surtout BrégeonPoli,1992).

Voilà des constatations bien triviales mais qui invitent à nuancer sensiblement les approches dénonçant, sur un mode volontiers justicier, la facticité des identités. Si les appartenances sont mouvantes et négociables, et non stables et à prix fixe, leurs variations demeurent tributaires des limites habituelles d'une transaction : on ne peut négocier que ce que l'on est, ce que l'on sait, ce que l'on a. Chacun fait quotidiennement, et parfois dramatiquement, l'expérience de ces limites de la négociation! Au bout du compte - et pour synthétiser un point de vue qui concilie approches -etic, -emic et contextualiste - on pourrait avancer la proposition suivante : à l'intérieur de "nébuleuses » culturelles produites par l'histoire, les groupes opèrent des choix d'identification, variables en nature, en intensité et en niveau, qui mettent en jeu, entre autres, leurs rapports avec la société englobante et ses institutions (voir Bromberger, Collomp et Centlivres, 1989).

Si le repérage, la production, l'affirmation, la perception des identités ont fait l'objet de nombreuses études ponctuelles, l'ethnologie de la France s'est, en revanche et paradoxalement, beaucoup moins interrogée sur les effets sociaux que produit la conscience d'une commune appartenance. Les comportements de solidarité, de fidélité, de »loyauté« que suscite le sentiment d'une identité partagée mériteraient des analyses attentives. De même les situations d'effervescence collective, où "l'on s'atteste à soi-même et l'on atteste à autrui que l'on fait partie du même groupe » (Durkheim, 1990 : 331), ont été scrutées en amont - à travers leur organisation -, dans l'instant - à travers les comportements des acteurs -, plus rarement en aval - à l'écho de leur résonance à court et à long termes. Ces moments festifs ou solennels (spectacles sportifs, célébrations, commémorations...) consacrent-ils seulement les identités ou peuvent-ils - et à quelles conditions ?- inaugurer des transformations du réel, autrement dit des « conquêtes » 22 ? L'examen de ces situations singulières, où se met

22 Sous le terme "conquêtes" Dayan (1990 : 22) désigne des événements ritualisés qui "réactivent des aspirations étouffées et déclenchent des mouvements fondateurs d'un nouveau rapport d'une société à son histoire". Sur la dynamique de ces événements spectaculaires qui peuvent entraîner des métamorphoses voir Bromberger (1990). 
en scène l'appartenance collective, permettrait d'éclairer d'un jour nouveau la dynamique des événements ritualisés et de mesurer à ses effets la pertinence du concept d’identité.

\section{Bibliographie}

\section{$\underline{\text { Retour à la table des matières }}$}

AGULHON, M., 1989. " La fabrication de la France, problèmes et controverses », in : L'autre et le semblable, Paris, Presses du C.N.R.S, pp. 109-120.

AUBRAC (L'), étude ethnologique, linguistique, agronomique et économique d'un établissement humain, 1972-1986, Paris, C.N.R.S, $6 \mathrm{t}$.

BEAUD, S. et NOIRIEL, G. " L'immigration dans le football », Vingtième Siècle, 26, av.-juin, pp. 83-96.

BELMONT, N., 1981. «Le problème du découpage régional dans l'oeuvre d'Arnold Van Gennep: pays et zones folkloriques », Le Monde alpin et rhodanien, 1, pp. 19-22.

BOZON, M. et THIESSE, A.-M., 1985. « Le donjon, le grenier et le jardin. La recomposition de l'identité culturelle et sociale du Valois », Terrain, 5, oct., pp. 6-15.

BREGEON-POLl, B., 1992, Crèche, 13 desserts, pastorale: la tradition en question, Mémoire de maîtrise, Université de Provence, Département d'Ethnologie, Aix-en-Provence.

BROMBERGER, C., 1987, «Du grand au petit. Variations des échelles et des objets d'analyse dans l'histoire récente de l'ethnologie de la France », in Ethnologies en miroir. La France et les pays de langue allemande (I. Chiva et U. Jeggle, éd.), Paris. Maison des Sciences de l'Homme, pp. 67-94.

BROMBERGER, C., 1989, «Ethnographie », in : BERTRAND, R., BROMBERGER, C. et al., Provence, Paris, Christine Bonneton, pp. 85-249. 
BROMBERGER, C., 1990, « Paraître en public. Des comportements routiniers aux événements spectaculaires », Terrain, 15, oct. 1990, pp. 5-12.

BROMBERGER, C., 1992a, «Ecomusées et espaces bâtis. Des formes architecturales aux significations culturelles ", in : Territoires de la mémoire (M. Augé, éd.), Thonon-les-Bains, L’Albaron, pp. 6789.

BROMBERGER, C, 1992b " Le football met à nu les antagonismes majeurs de nos sociétés », Le Monde Diplomatique, juin 1992, pp. 10-11.

BROMBERGER, C., DOSSETTO, D., et SCHIPPERS, T., 19821983, «L'ethnocartographie en Europe : coups d'oeil rétrospectifs et questions ouvertes ", in : "L'ethnocartographie en Europe », Technologies, Idéologies, Pratiques, IV, 1-4, pp. 15-40.

BROMBERGER, C., COLLOMP, G. et CENTLIVRES, P., 1989, « Entre le local et le global : les figures de l'identité », in : L'Autre et le semblable, Paris, Presses du C.N.R.S., pp. 137-146.

BURGUIERE, A., 1975, Bretons de Plozévet, Paris, Flammarion.

CHIVA, 1., 1990, « Le patrimoine ethnologique. L'exemple de la France », Encyclopaedia Universalis, vol. 24 (symposium), pp. 229241.

CUISENIER, J., 1982-1983, « La cartographie ethnographique au Musée National des Arts et Traditions populaires ", in : L'ethnocartographie en Europe, pp. 191-226.

CUISENIER, J., 1991, " Que faire des arts et traditions populaires ? », Le débat, mai-août, pp. 150-165.

DALLA BERNARDINA, S., 1989, « L’invention du chasseur écologiste. Un exemple italien », Terrain, 13, oct. 1989, pp. 130- 139.

DAYAN, D., 1990, "Présentation du pape en voyageur. Télévision, expérience rituelle, dramaturgie politique », Terrain, 15, oct. 1990, pp. 13-28.

DENIS, M.-N., 1987, « Ethnologie en Alsace (1830-1980) : Identité politique et politique d'identité », Anthropologie sociale et ethnologie de la France (colloque du Centre d'Ethnologie Française et du 
Musée National des Arts et Traditions Populaires (Communications, t. 1).

DURKHEIM, E., 1990, (rééd.), Les formes élémentaires de la vie religieuse, Paris, Presses Universitaires de France.

EHRENBERG, A., 1991, Le culte de la performance, Paris, Calmann-Lévy. FAURE, C.. 1989, Le projet culturel de Vichy, Lyon, Presses Universitaires de Lyon.

GUILCHER, J.-M., 1984, La tradition de danse en Béarn et pays basque français, Paris, Maison des Sciences de l'Homme.

ISAMBERT, F.-A et TERRENOIRE, J.-P., Atlas de la pratique religieuse des catholiques en France, Paris, C.N.R.S. et Presses de la Fondation nationale des sciences politiques.

KRÜGER, F., 1950, Géographie des traditions populaires en France, Mendoza, d'Accurzio impresor, 2 vol.

LAUTMAN, F., 1988, « La Sainte Estelle à Saint-Junien, fête emblématique et enjeu politique », Ethnologie Française, 3. juil.-sept, pp. 298- 302.

MAGET, M., 1955, « Remarques sur le village comme cadre des recherches anthropologiques », Bulletin de psychologie, VIII, 7-8, pp. 375-382.

OLLIVIER, E., 1987. "Les monuments historiques demain... ", Terrain, 9, oct., pp. 124-127.

RAPHAEL, F. et HERBERICH-MARX, G., 1991, Mémoire plurielle de l'Alsace. Grandeurs et servitudes d'un pays des marges, Strasbourg, Publications de la Société savante d'Alsace et des régions de l'Est.

RAVIS-GIORDANI, G. 1989, "Un peuple sans identité ou une identité sans peuple, La Corse à la croisée des chemins ", La pensée, mars-avril, pp. 329-339.

REDFIELD, R.. 1960, The little Community and Peasant Society and Culture, Chicago et Londres, The University of Chicago Press.

RENAN, E., 1947, "Qu'est-ce qu'une nation ? », in : Oeuvres complètes, Paris, Calmann-Lévy, t. l, pp. 887-906 (texte d'une conférence prononcée en 1882). 
SCHNAPPER, D., 1991, La France de l'intégration. Sociologie de la nation en 1990, Paris, Gallimard.

SIEBURG, F., 1930, Dieu est-il français ?, Paris, Grasset.

THIESSE, A.-M., 1988, «Le mouvement littéraire régionaliste », Ethnologie Française, 3, juil.-sept., pp. 220-232.

THIESSE, A.-M., 1991, Écrire la France. Le mouvement littéraire régionaliste de langue française entre la belle Époque et la Libération, Paris, Presses Universitaires de France.

\section{Pour citer cet article}

Christian Bromberger, «L'ethnologie de la France et le problème de l'identité ", Civilisations [En ligne], vol. 42, no 2, 1993, mis en ligne le 30 décembre 1996, Consulté le 27 mars 2012. URL.

\section{Fin du texte}

